\title{
Numerical simulations for fractional variable--order difference eigenfunctions
}

\author{
Piotr Oziabło
}

\author{
Faculty of Computer Science \\ Bialystok University of Technology, Wiejska 45A, Biatystok, Poland \\ (e-mail: p.oziablo@student.pb.edu.pl)
}

Keywords: Difference equations; Dynamic systems; Eigenfunction; Fractional variable-order.

\section{INTRODUCTION AND PRELIMINARIES}

Recently fractional calculus is under strong attention as useful tool in modelling. In many cases fractional derivatives and difference operators proved their usefulnes and effectiveness in describing many real-life processes and phenomenas. For a review of theory and applications of fractional calculus, we refer the reader to (Hilfer, 2000; Kaczorek, 2009; Mozyrska \& Wyrwas, 2015; Ostalczyk, 2016; Podlubny, 1999). For variable-order applications the reader can see more in Mozyrska \& Ostalczyk (2017). In the paper we investigate discrete time operators with variable orders. We define variable-, fractional order backward difference of the Grünwald-Letnikov-type which means that the order is a single-variable, positive-valued function. Our goal is to start investigations of fitting data for noised eigenvalue function for initial value problem for fractional difference with variable-order.

Definition 1. For $k, l \in \mathbb{Z}$ and a given order function $\nu(\cdot)$ we define the oblivion function, as a discrete function of two variables, by its values $a^{[\nu(l)]}(k)$ given as

$a^{[\nu(l)]}(k)=(-1)^{k} \frac{\nu(l)[\nu(l)-1] \cdots[\nu(l)-k+1]}{k !}$, for $k>0$

and $a^{[\nu(l)]}(k)=0$ for $k<0, a^{[\nu(l)]}(0)=1$.

Formula (1) in Definition 1 is equivalent to the following recurrence with respect to $k \in \mathbb{N}$ :

$$
\begin{aligned}
& a^{[\nu(l)]}(0)=1, \\
& a^{[\nu(l)]}(k)=a^{[\nu(l)]}(k-1)\left[1-\frac{\nu(l)+1}{k}\right] \text { for } k \geqslant 1 .
\end{aligned}
$$

Definition 2. Let $f$ be a discrete-variable bounded real valued function. The Grünwald-Letnikov variable-, fractionalorder backward difference (GL-VFOBD) with an order function $\nu: \mathbb{Z} \rightarrow \mathbb{R}_{+} \cup\{0\}$ of function $x(\cdot)$ is defined as a finite sum

\footnotetext{
* The work was supported by Polish founds of National Science Center, granted on the basis of decision DEC-2016/23/B/ST7/03686 and COST Action CA15225.
}

$$
\begin{aligned}
&\left(\Delta^{[\nu(k)]} x\right)(k)=\sum_{i=0}^{k} a^{[\nu(k)]}(i) x(k-i)= \\
& {\left[\begin{array}{lll}
1 & a^{[\nu(k)]}(1) \cdots & a^{[\nu(k)]}(k)
\end{array}\right]\left[\begin{array}{c}
x(k) \\
x(k-1) \\
\cdots \\
x(1) \\
x(0)
\end{array}\right] }
\end{aligned}
$$

Let

$$
\mathbf{A}^{[\nu(k)]}:=\left[\begin{array}{cccc}
1 & a^{[\nu(k)]}(1) & \cdots & a^{[\nu(k)]}(k) \\
0 & 1 & \cdots & a^{[\nu(k-1)]}(k-1) \\
\vdots & \vdots & & \vdots \\
0 & 0 & \cdots & 1
\end{array}\right] .
$$

We consider the simple equation with variable-order for $k_{0}=0$.

$$
\left(\Delta^{[\nu(k)]} x\right)(k)=\lambda x(k-1), k \geq 1
$$

with initial condition $x(0)=x_{0}$ and constant coefficient $\lambda \in \mathbb{R}$. Then we can solve equation (5) by recurrence

$$
x(k)=-\sum_{i=1}^{k} a^{[\nu(k)]}(i) x(k-i)+\lambda x(k-1), k \geq 1 .
$$

Instead of working with recurrence we can use matrix form of defined matrices $\mathbf{A}_{k}^{[\nu(k)]}$. Moreover, let us use the following notation

$$
\mathbf{x}(k)=\left[\begin{array}{c}
x(k) \\
x(k-1) \\
\vdots \\
x(1) \\
x(0)
\end{array}\right]
$$

Then, equation (5) can be written in the matrix form

$$
\mathbf{A}^{[\nu(k)]} \mathbf{x}(k)=\left[\begin{array}{c}
\lambda \mathbf{x}(k-1) \\
x(0)
\end{array}\right], \quad k \geq 1
$$

and its solution looks like the series of algebraic solutions:

$$
\mathbf{x}(k)=\left(\mathbf{A}^{[\nu(k)]}\right)^{-1}\left[\begin{array}{c}
\lambda \mathbf{x}(k-1) \\
x(0)
\end{array}\right] \text {. }
$$




\section{FRACTIONAL VARIABLE-ORDER EQUATIONS APPROXIMATION}

Fitting experimental data is one of the most important issue for modeling. Efficient methods of finding fractional order equations which could describe real life phenomena would help us to develop better and more accurate mathematical models. In the first stage of our research we are trying to find a way to determine a value of $\lambda$ knowing the order function. Additionally, we add noise signal to the data sets, which are used as an input data for our calculation to simulate "real life" measurement data. This approach emulates finding mathematical model which describes some real process.

We calculate GL-VFOBD values based on (8) matrix definition for different order functions and the different levels of noise. At the beginning we are trying to estimate $\lambda$ using Mean Squared Error as a qualitative criterion of the estimation. Let $\mathbf{y}_{k}=\mathbf{x}(k)+\epsilon$, where $\epsilon \sim N(0, \sigma)$ is added noise. We assume, that we know order function, so we know also matrices given by formula (4). Then, the simplest criterion to fit $\lambda$ is to minimize the following function

$$
S_{k}(\lambda):=E_{k}^{T} E_{k}
$$

where

$$
E_{k}=\mathbf{y}_{k}-\lambda\left(\mathbf{A}^{[\nu(k)]}\right)^{-1}\left[\begin{array}{c}
\mathbf{y}_{k-1} \\
y_{0}
\end{array}\right]
$$

The formula is slightly different as in equations (8), which gives us simpler formulation of the minimizer.

Then, $\lambda_{0}$ minimizing $S_{k}$ for the set of $k+1$ values is given by the formula

$$
\lambda_{0}=\frac{1}{2 d}\left(\mathbf{y}_{k}^{T} A^{-1}\left[\begin{array}{c}
\mathbf{y}_{k-1} \\
y_{0}
\end{array}\right]+\left[\mathbf{y}_{k-1}^{T} y_{0}\right]\left(A^{T}\right)^{-1} \mathbf{y}_{k}\right),
$$

where

$$
d=\left[\mathbf{y}_{k-1}^{T} y_{0}\right]\left(A^{T}\right)^{-1} A^{-1}\left[\begin{array}{c}
\mathbf{y}_{k-1} \\
y_{0}
\end{array}\right]
$$

and $A=\mathbf{A}^{[\nu(k)]}$

Then, theoretical values $\hat{\mathbf{y}}_{k}$ we have from formula:

$$
\hat{\mathbf{y}}_{k}=\left(\mathbf{A}^{[\nu(k)]}\right)^{-1}\left[\begin{array}{c}
\lambda_{0} \mathbf{y}_{k-1} \\
y_{0}
\end{array}\right] \text {. }
$$

In figures 1 and 2 we show some examples of order functions, noised data and the theoretical data from approximation given by (10). During our research we found out that $\lambda$ estimation quality may be different for given order function even for the same level (variance and mean value) of noise. For some of the random noise values $\lambda$ was not estimated properly (estimation error was significant). This was particularly evident when added noise values were high.

\section{CONCLUSION}

In future plans we are going to extend our investigations to matrix-eigenvalue form and also for situations, where we do not know parameters of order-function. Moreover, we are expecting some results in fitting real data by fractional models with variable order.
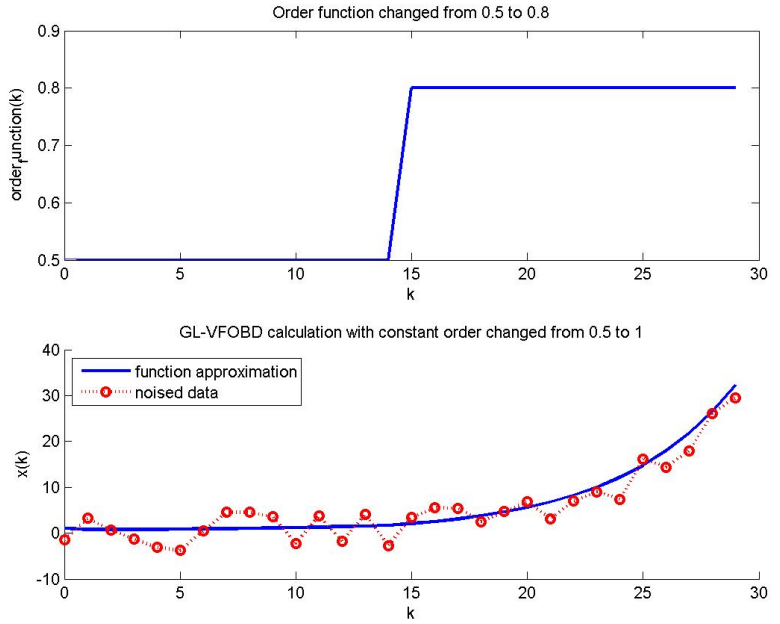

Fig. 1. Order function $\nu(\cdot)$ changed values from 0,5 to 0,8 , original $\lambda=0.3$, approximated $\lambda=0.3035$
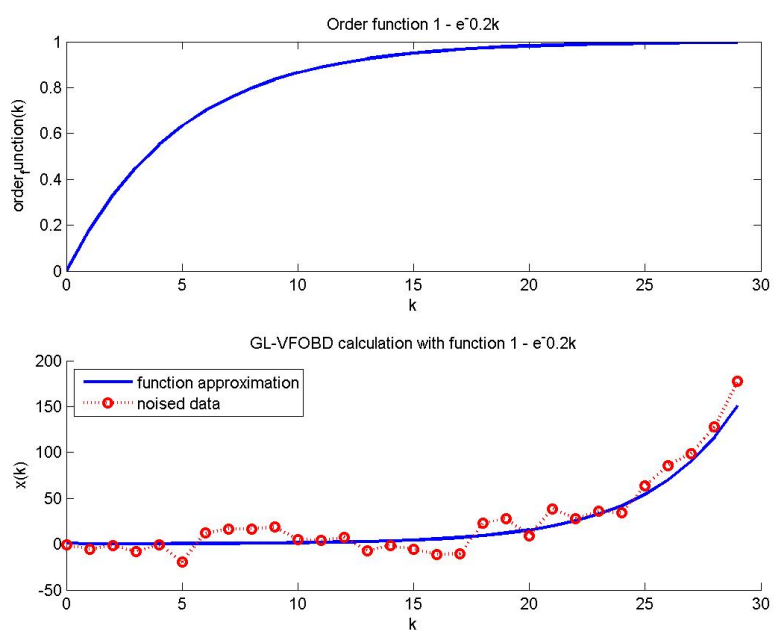

Fig. 2. Order function $\nu(k)=1-e^{-0,2 k}$, original $\lambda=0.3$, approximated $\lambda=0.2923$

\section{REFERENCES}

Hilfer, R. (2000). Applications of Fractional Calculus in Physics. World Scientific Publishing Company, Singapore.

Kaczorek, T. (2009). Fractional positive linear systems. Kybernetes, 38(7/8):1059-1078.

Mozyrska, D. \& Wyrwas, M. (2015). The Z-transform method and delta type fractional difference operators. Discrete Dynamics in Nature and Society, 2015:25 pages.

Mozyrska, D. \& Ostalczyk, P. (2017). Generalized Fractional-Order Discrete-Time Integrator. Complexity, Volume 2017, Article ID 3452409, 11 pages.

Ostalczyk, P. (2016). Discrete fractional calculus. Applications in control and image processing. World Scientific Publishing Co Pte Ltd, vol. Series in Computer Vision - Vol. 4.

Podlubny, I. (1999). Fractional Differential Equations. Mathematics in Sciences and Engineering. 198. Academic Press, San Diego. 